Palavras chave:

Deflexão

Ensaios mecânicos

Dimensionamento de peças

Histórico:

Recebido 03/09/2014

Aceito 15/1 I/2015

Keywords:

Deflection

Mechanical testing

Dimensioning of parts

Correspondência: valeriapazetto@gmail.com
Valéria Maria de Figueiredo Pazetto', Cláudio Henrique Soares Del Menezzi', Joaquim Carlos Gonçalez'

\section{EFEITO DA ESPESSURA E DO VÃO NA DEFORMAÇÃO EM FLEXÃO DE PAINÉIS DE MADEIRA}

RESUMO: Este trabalho teve como objetivo avaliar a correlação entre as propriedades físicas e mecânicas de painéis de MDF (Medium Density Fiberboard) e de MDP (Medium Density Particleboard) utilizados na fabricação de móveis. Para análise dos painéis foram determinadas as propriedades físicas de densidade, e teor de umidade e as propriedades mecânicas em flexão estática (MOE e MOR). O experimento foi realizado com amostras de painéis, em três espessuras ( $15 \mathrm{~mm}, 18 \mathrm{~mm}$ e $25 \mathrm{~mm}$ ) e três vãos considerando a relação $L / h$ (vão dividido pela espessura) de 30,35 e 40, totalizando nove tratamentos para cada tipo de painel, com três repetições. Essas amostras também foram submetidas a ensaios não destrutivos de flexão, com cargas equivalentes a $25 \mathrm{~g} \cdot \mathrm{cm}^{-2}$ uniformemente distribuídas, em condições climatizadas (temperatura $20^{\circ} \mathrm{C} \pm \mathrm{I}$ e umidade $65 \% \pm \mathrm{I}$ ). A deformação final com a carga foi registrada após uma semana registrando-se ainda a deformação residual após a retirada da carga. Foi feita uma análise estatística dos resultados, onde se avaliou o efeito dos tratamentos relativos ao vão e a espessura. Uma análise das médias evidenciou diferenças significativas entre os valores obtidos em cada espessura. $O$ vão demonstrou ter maior influência na deformação dos painéis do que a espessura. Entre as dimensões das amostras avaliadas a melhor relação encontrada foi $\mathrm{L} / \mathrm{h}=30$.

\section{EFFECT OF THICKNESS AND SPAN IN BENDING DEFORMATION OF WOOD PANELS}

ABSTRACT: This study aimed to evaluate the correlation between the physical and mechanical properties of MDF (Medium Density Fiberboard) and MDP (Medium Density Particleboard) used in furniture manufacturing. The physical properties density and moisture content and the mechanical properties static bending (MOE and MOR) were determined. The experiment was made with three panel thicknesses $(15 \mathrm{~mm}, 18 \mathrm{~mm}$ and $25 \mathrm{~mm}$ ) and three different spans with relationship L/h (span divided by thickness) of 30 , 35 and 40 totaling nine treatments for each type of panel with three replications. These samples were subjected to nondestructive bending test, with loads equivalent to $25 \mathrm{~g} \cdot \mathrm{cm}^{-2}$ uniformly distributed in conditioned environment (temperature $20^{\circ} \mathrm{C} \pm \mathrm{I}$, humidity $65 \%$ $\pm \mathrm{I}$ ). The final deformation load was recorded after a week, and the residual deformation, after removal of the load, was also registered. Statistical analysis of the results evaluated the effect of treatments relative to the span and thickness. The analysis showed significant differences between the results for each thickness, however, the span demonstrated greater influence than the thickness. Among the sample sizes evaluated the best value found was $L / h=30$.
DOI: 


\section{INTRODUÇÃO}

A utilização dos painéis de madeira reconstituída em substituição à madeira maciça vem sendo cada vez mais frequente, tanto na fabricação de móveis como na construção civil. Esse crescimento se deve, principalmente, a fatores como a escassez de madeira maciça, a estabilidade dimensional dos painéis, a maior uniformidade de suas propriedades e a evolução tecnológica na fabricação desses produtos, permitindoIhes melhor usinagem e acabamento. Além desses fatores, há o fato da aceitação por parte dos consumidores como um material sustentável, já que é fabricado a partir de madeira de reflorestamento, diminuindo a demanda de recursos nativos (DA ROSA et al., 2007).

Segundo Bodig e Jayne (1993), a resistência à deformação acontece na medida em que o material é comprimido, fletido ou distorcido sob o efeito da carga/ força, podendo ocorrer a deformação elástica (mudanças de forma instantâneas) ou a deformação reológica (dependendo do tempo de aplicação).

A madeira e seus derivados são materiais viscoelásticos e caracterizam-se por apresentar uma resposta de deformação em função do tempo para uma determinada tensão aplicada, isto significa que o comportamento do material é dependente do tempo, portanto, em um instante qualquer, sob carga, seu desempenho será em função do esforço acumulado ao longo do tempo, causado pela fluência. Quando exposta à tensão constante a deformação aumentará com o tempo, podendo causar a ruptura do material (FRIHART, 2005).

Desta forma, as variações causadas pelas dimensões e formas dos corpos-de-prova sobre as propriedades de resistência da madeira podem ser expressas pela relação comprimento/aresta da seção transversal ou altura $(\mathrm{L} / \mathrm{h})$. A relação $\mathrm{L} / \mathrm{h}$ ideal para teste de compressão paralela às fibras é aproximadamente igual a quatro. Já em ensaios de flexão estática a relação $\mathrm{L} / \mathrm{h}$ ideal chega a ser superior a vinte, pois a partir deste ponto é atribuída uma resistência máxima e constante à madeira (MORESCHI, 2005).

Tem-se observado em ensaios de laboratório, que os painéis sofrem deformações excessivas nas superfícies horizontais sob carregamento, logo, a distância entre os apoios e a profundidade da superfície deve estar dimensionada de acordo com a carga a ser utilizada. Desta forma, as dimensões das peças determinam o tipo e a espessura do material a ser usado.

Essa tendência à deformação em flexão, ao longo do tempo sob carregamento, motivou a realização deste estudo, que tem como objetivo analisar a correlação entre as propriedades físicas e mecânicas dos painéis, e a espessura e o vão das amostras na deformação em flexão de painéis de MDF e de MDP, visando sua aplicação em projetos de móveis que possuem superfícies horizontais de carregamento.

\section{MATERIAIS E MÉTODOS}

\section{Materiais}

Foram utilizados três painéis de MDF (Medium Density Fiberboard) e três painéis de MDP (Medium Density Particleboard) com dimensões de $1830 \times 2750$ $\mathrm{mm}$ e espessuras de $15 \mathrm{~mm}$, $18 \mathrm{~mm}$ e $25 \mathrm{~mm}$. Os painéis de MDF foram produzidos a partir de madeira de pinus e resina à base de ureia-formaldeído. Os painéis de MDP foram produzidos a partir de partículas de eucalipto e resina à base de ureia-formaldeído.

\section{Ensaios de flexão estática}

Foram realizados os ensaios de flexão estática a partir de 4 (quatro) amostras retiradas de cada uma das espessuras dos painéis de MDF e de MDP, totalizando 24 corpos-de-prova (CPs). O vão utilizado foi de 24 vezes a espessura nominal do painel e a largura de 76 $\mathrm{mm}$. O ensaio for conduzido segundo a norma ASTM D-1037:99, com a aplicação da carga no centro do vão, a uma velocidade uniforme, através de um bloco de carregamento arredondado. As amostras foram colocadas em câmara climatizada $\left(20^{\circ} \mathrm{C} \pm \mathrm{I}\right.$ e $65 \%$ $\pm \mathrm{I}$ ) onde permaneceram até atingir a umidade de equilíbrio (12\%). As amostras foram medidas e pesadas para determinação da densidade aparente dos painéis. Os ensaios foram feitos no Laboratório de Produtos Florestais do Serviço Florestal Brasileiro em máquina universal INSTRON, com capacidade de aplicação de carga de vinte toneladas. A partir desse ensaio foram obtidos o módulo de elasticidade (MOE), módulo de ruptura (MOR), a carga no limite proporcional (Plim) e deformação no limite proporcional (Dlim) dos painéis.

\section{Ensaios de flexão de planos horizontais}

Os ensaios de flexão de planos horizontais avaliaram a deformação de painéis de MDF e MDP, sob efeito de carga por um período de sete dias. A avaliação do desempenho dos painéis foi estabelecida pela norma NBR | 396 I:20 I0, que admite uma deformação de 0,5\% 
da distância entre os apoios com o plano carregado e $0,1 \%$ da distância entre os apoios após a remoção da carga. Isso pressupõe que, num vão de $1000 \mathrm{~mm}$ a deformação com carga permitida seria de $5,0 \mathrm{~mm}$ e residual de $1,0 \mathrm{~mm}$. Para tanto, foram determinadas as relações L/h de 30, 35 e 40, ou seja, a razão entre o vão (medida entre os apoios) e a espessura dos painéis. De cada chapa foram retirados 9 (nove) corpos-de-prova (CPs) com $400 \mathrm{~mm}$ de largura e vãos baseados nas relações $L / h$ pré-determinadas, conforme Tabela I. Essas dimensões de vão foram escolhidas porque representam as variações nas dimensões da maioria das prateleiras utilizadas em mobiliário. A quantidade total de CPs foi de 54, sendo 27 de MDF e 27 de MDP, uma vez que foram estudadas três espessuras, em três vãos distintos e três repetições de cada ensaio (Tabela I).

Tabela I Dimensões dos vãos das amostras dos ensaios de flexão.

Table I Span dimensions of the samples of bending tests.

\begin{tabular}{cccc}
\hline \multirow{2}{*}{ Relação L/h } & \multicolumn{3}{c}{ Vão dos painéis por espessura } \\
\cline { 2 - 4 } & $15 \mathrm{~mm}$ & $18 \mathrm{~mm}$ & $25 \mathrm{~mm}$ \\
\hline 30 & 450 & 540 & 750 \\
35 & 525 & 630 & 875 \\
40 & 600 & 720 & 1000 \\
\hline
\end{tabular}

Foram retiradas amostras de cada painel, para a determinação de sua densidade aparente. $O$ teor de umidade foi determinado pelo método gravimétrico, e as amostras foram pesadas e mantidas em estufa a $103^{\circ} \mathrm{C}$ $( \pm 2)$ por cinco dias até peso constante.

A duração de cada ensaio de flexão foi de uma semana, a carga aplicada em cada CP foi de $25 \mathrm{~g} \cdot \mathrm{cm}^{-2}$. A carga uniformemente distribuída foi aplicada por pesos graduados. A deformação em flexão foi medida em milímetro no ponto médio entre os apoios, por relógios comparadores analógicos: antes da aplicação da carga, ao final do ensaio (sete dias) com o plano carregado, e após a remoção da carga (deformação residual). Foram avaliados também os valores da deformação final e residual em relação à espessura e os valores da deformação final e residual relativos ao vão das amostras.

\section{Análise estatística}

Foi utilizado um delineamento estatístico do tipo fatorial completo $3 \times 3$, que corresponde às três espessuras dos painéis e as três relações $L / h$ totalizando nove tratamentos com três repetições para cada tratamento. $O$ delineamento estatístico foi desenvolvido para se avaliar o efeito do vão e da espessura dos painéis sobre as variáveis numéricas, além da comparação entre os resultados dos ensaios realizados em painéis de MDF e MDP. Os dados foram submetidos à análise de variância e teste de médias (Tukey a 5\% de significância). A análise estatística foi feita com o auxílio do software SPSS Statistical Package for the Social Sciences (Tabela 2).

Tabela 2 Planejamento doexperimento pelonúmero de tratamentos. Table 2 Planning of the experiment by the number of treatments.

\begin{tabular}{ccccc}
\hline Tratamento & $\begin{array}{c}\text { Espessura } \\
(\mathrm{mm})\end{array}$ & $\begin{array}{c}\text { Relação } \\
\text { L/h }\end{array}$ & $\begin{array}{c}\text { Carga } \\
\text { distribuída } \\
(\mathrm{kg})\end{array}$ & $\begin{array}{c}\mathrm{N}^{\circ} \text { de } \\
\text { repetições }\end{array}$ \\
\hline T1 & 15 & 30 & 45 & 3 \\
T2 & 15 & 35 & 52,5 & 3 \\
T3 & 15 & 40 & 60 & 3 \\
T4 & 18 & 30 & 54 & 3 \\
T5 & 18 & 35 & 60 & 3 \\
T6 & 18 & 40 & 72 & 3 \\
T7 & 25 & 30 & 75 & 3 \\
T8 & 25 & 35 & 87,5 & 3 \\
T9 & 25 & 40 & 100 & 3 \\
\hline
\end{tabular}

\section{RESULTADOS E DISCUSSÃO}

\section{Caracterização dos painéis de MDF e de MDP}

A densidade aparente ( $12 \%$ de umidade) dos painéis de MDF variou entre 0,64 e $0,70 \mathrm{~g} \cdot \mathrm{cm}^{-3}$ e o teor de umidade entre $8,4 \%$ e $9,5 \%$. Para os painéis de MDP, a densidade encontrada foi entre 0,58 e $0,63 \mathrm{~g} \cdot \mathrm{cm}^{-3}$, a umidade variou entre 10\% e 10,6\% (Figura I).

Apesar de apresentarem valores numéricos semelhantes os painéis apresentaram diferenças significativas nas densidades entre as espessuras

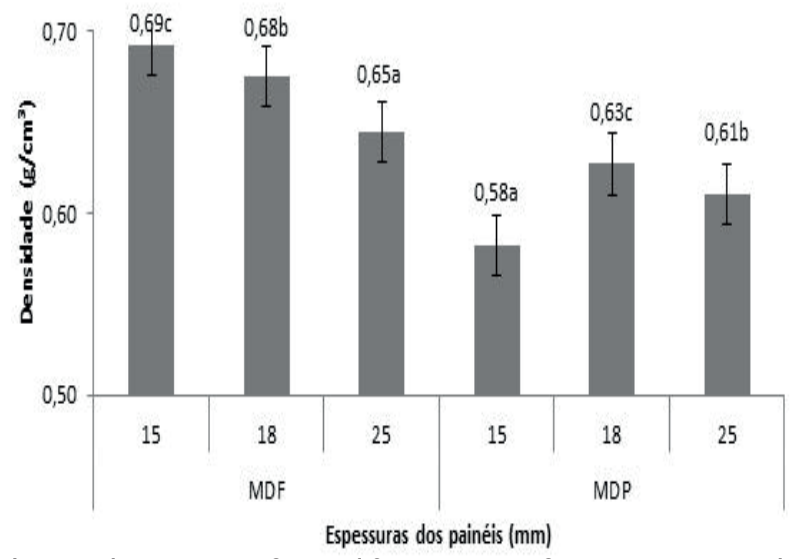

Letras distintas significam diferenças significativas no teste de Tukey a $5 \%$ de significância.

Figura I Densidade de cada espessura de MDF e MDP.

Figure I Density of each thickness of MDF and MDP. 
analisadas. Observou-se que a densidade encontrada nos painéis de MDF foi superior aos dos painéis de MDP. Isso se deve ao fato do MDF possuir menos espaços vazios, existindo assim, maior área de contato entre as fibras de madeira do que no MDP.

\section{Ensaios de flexão estática}

\section{Carga e deformação no limite proporcional}

A análise estatística detectou a existência de diferenças significativas tanto na carga, como na deformação dos painéis. Os maiores valores de carga e deformação no limite proporcional estavam diretamente relacionados com a espessura dos painéis, ou seja, os painéis de maior espessura obtiveram valores mais altos de carga e deformação no limite proporcional. Observou-se também, que os valores de deformação do MDF foram maiores do que os de MDP com mesma espessura (Figura 2).

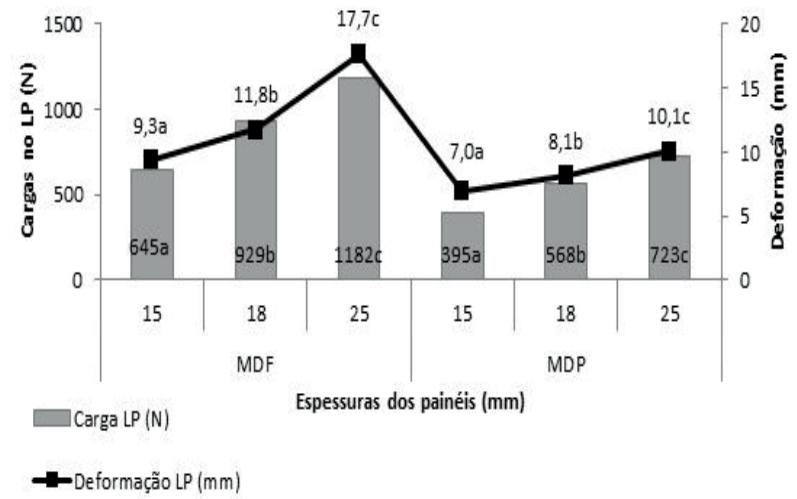

Letras distintas significam diferenças significativas no teste de Tukey a 5\% de significância.

Figura 2 Valores de carga e deformação no limite proporcional por espessura de painéis.

Figure 2 Values of load and deformation at proportional limit for panel thickness.

Módulo de Ruptura e Módulo de Elasticidade

Os valores de MOE dos painéis de MDF não apresentaram relação com a densidade encontrada, uma vez que, o painel de $15 \mathrm{~mm}$ apresentou a maior densidade, mas não o maior valor do MOE. Já os painéis de MDP apresentaram relação entre MOE e densidade, tendo o painel de $18 \mathrm{~mm}$ apresentado a maior densidade e o maior valor do MOE (Figura 3).

Uma justificativa para esse comportamento do MDF, segundo Eleotério (2004), seria a influência de outros fatores nas propriedades de resistência do painel, além da densidade média. Como por exemplo,

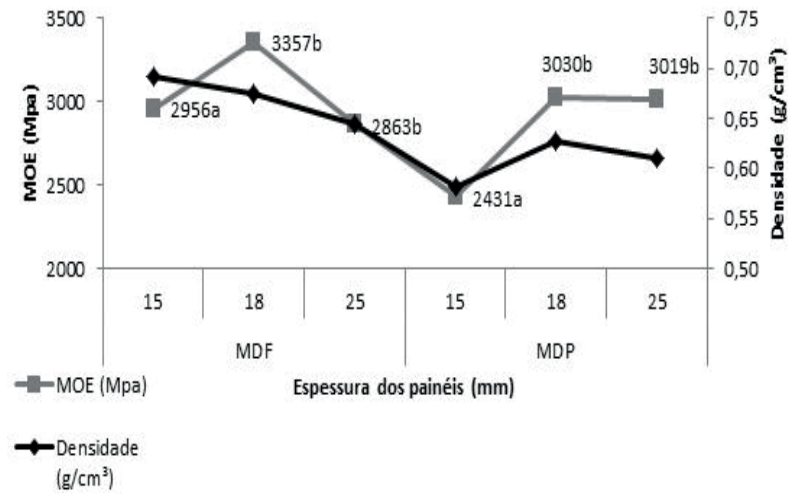

Letras distintas significam diferenças significativas no teste de Tukey a $5 \%$ de significância.

Figura 3 MOE e a Densidade de cada espessura de painel de MDF e MDP.

Figure $3 \mathrm{MOE}$ and Density of each panel thickness of MDF and MDP.

a densidade da matéria-prima, o comprimento das fibras, a densidade da camada externa do painel, teor de resina, teor de umidade do colchão e outras variáveis relacionadas ao desfibramento.

Outros fatores, como a razão de compactação, a geometria das partículas e o teor de resina utilizado, também não foram conhecidos, o que segundo Maloney (1993), também podem influenciar nas propriedades mecânicas dos painéis.

Conforme apresenta a Figura 4, os resultados do MOR também não apresentaram relação com os valores de densidade dos painéis. O MDF com espessura de 18 $\mathrm{mm}$ obteve os maiores valores de MOR, enquanto que a densidade maior foi encontrada nos painéis de $15 \mathrm{~mm}$. Já, no MDP os maiores valores de densidade foram encontrados nos painéis de $18 \mathrm{~mm}$, mas não houve diferenças significativas entre o MOR dos painéis de $18 \mathrm{~mm}$ e $25 \mathrm{~mm}$.

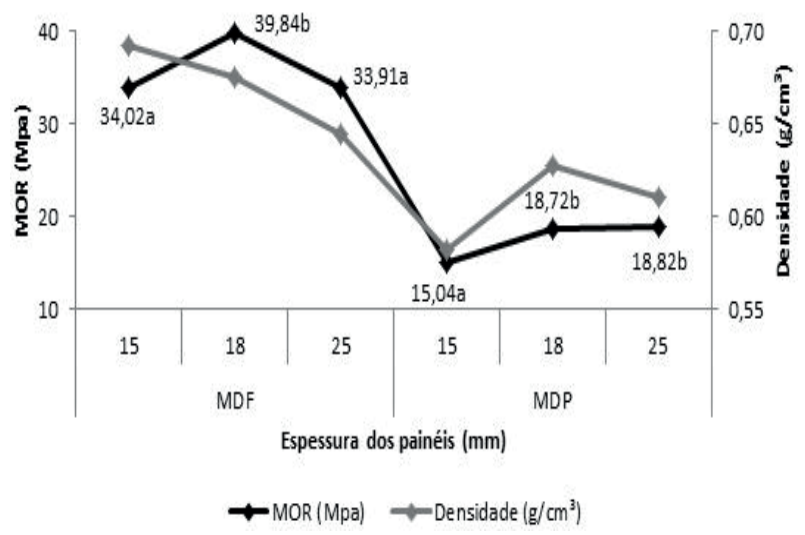

Letras distintas significam diferenças significativas no teste de Tukey a $5 \%$ de significância

Figura 4 Relação entre MOR e densidade de cada espessura de painéis de MDF e MDP.

Figure 4 Relation between MOR and Density of each panel thickness of MDF and MDP. 


\section{Ensaios de flexão em prateleiras}

A ANOVA (Análise de Variância) fatorial do experimento apontou que o efeito da espessura apresentou resultados significativos, nas deformações: final com carga, residual por espessura e na interação entre as variáveis dependentes: espessura e vão.

\section{Ensaios de flexão em MDF}

O teste de Tukey apresentou diferenças significativas no resultado da deformação dos painéis de MDF, com os valores encontrados de deformação divididos pela espessura $(\mathrm{mm} / \mathrm{mm})$. Isto mostra, que a maior deformação final com plano submetido à carga ocorreu nos tratamentos T3, T6 e T9, que correspondem à relação $\mathrm{L} / \mathrm{h}=40$. Já a menor deformação com carga ocorreu no tratamento T4 com espessura de $18 \mathrm{~mm}$ e vão com relação $\mathrm{L} / \mathrm{h}=$ 30. No entanto, após a retirada da carga a maior deformação residual ocorreu nos tratamentos T7, T8 e T9, ou seja, nos painéis com espessura de 25

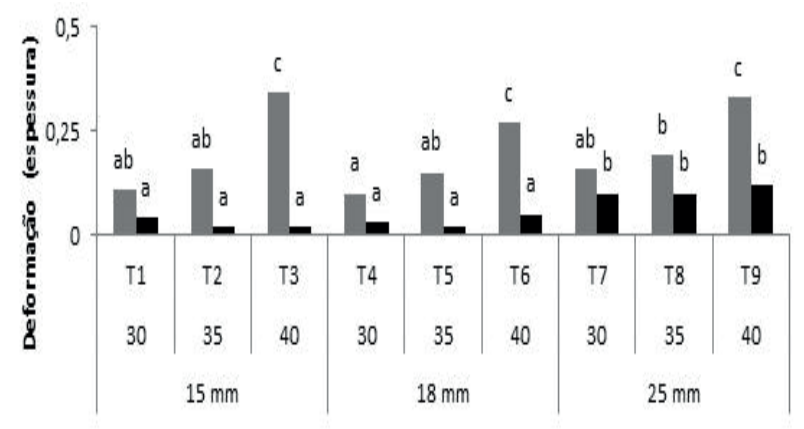

- Final c/ carga

- Residual

Letras distintas significam diferenças significativas no teste de Tukey a $5 \%$ de significância.

Figura 5 Valores médios da deformação final com carga e deformação residual relativos à espessura para os tratamentos com MDF.

Figure 5 Mean values of final deformation under load and residual deformation for each thickness for the treatments with MDF.

$\mathrm{mm}$, os demais tiveram deformações semelhantes, independente da relação L/h (Figura 5).

A Figura 6 mostra a deformação dos painéis de MDF divididos pelo vão. Onde a maior deformação com carga foi encontrada nos vãos com relação $L / h$ $=40$, e a menor na relação $L / h 30$ (tratamento T4).

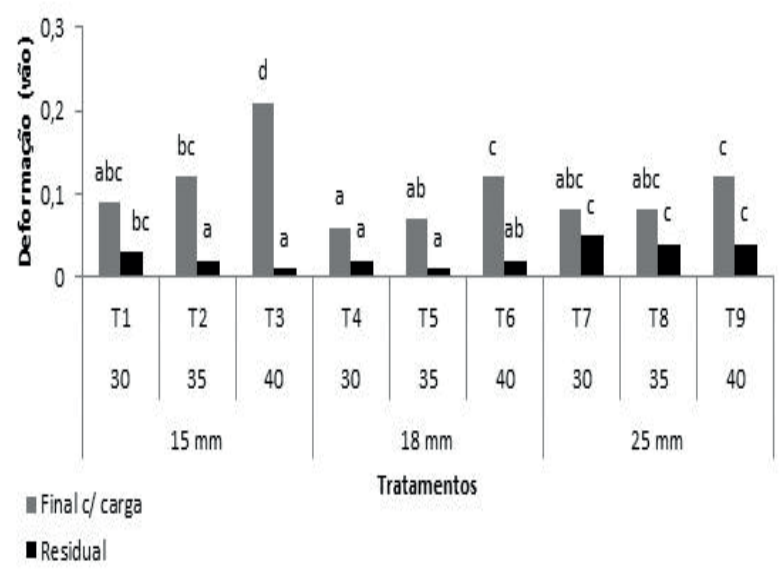

Letras distintas significam diferenças significativas no teste de Tukey a $5 \%$ de significância.

Figura 6 Valores médios da deformação final com carga e deformação residual relativos ao vão para os tratamentos com MDF.

Figure 6 Mean values of final deformation under load and residual deformation for each span for the treatments with MDF

\section{Ensaios de flexão em MDF}

Observando-se os valores da deformação relativa em função da espessura dos painéis de MDP, notase que a maior deformação final com carga foi registrada no tratamento T3, assim como a maior deformação residual. Os menores valores de deformação final com carga foram registrados nos tratamentos T4 e T7, e da deformação residual no tratamento TI. Desta forma, a deformação final com carga apresentou valores que se destacam mais que a deformação residual (Figura 7).

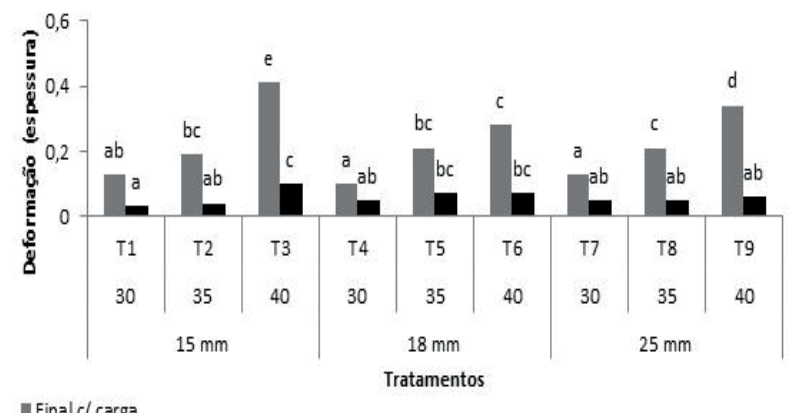

- Final c/ carga

- Residual

Letras distintas significam diferenças significativas no teste de Tukey a $5 \%$ de significância.

Figura 7 Valores médios da deformação final com carga e deformação residual relativos à espessura para os tratamentos com MDP.

Figure 7 Mean values of final deformation under load and residual deformation for each thickness for the treatments with MDP. 
Para os valores relativos ao vão (Figura 8), a maior deformação com carga foi encontrada no T9. E as menores deformações com carga e residual foram registradas nos tratamentos $\mathrm{T}$ I, T2 e T4.

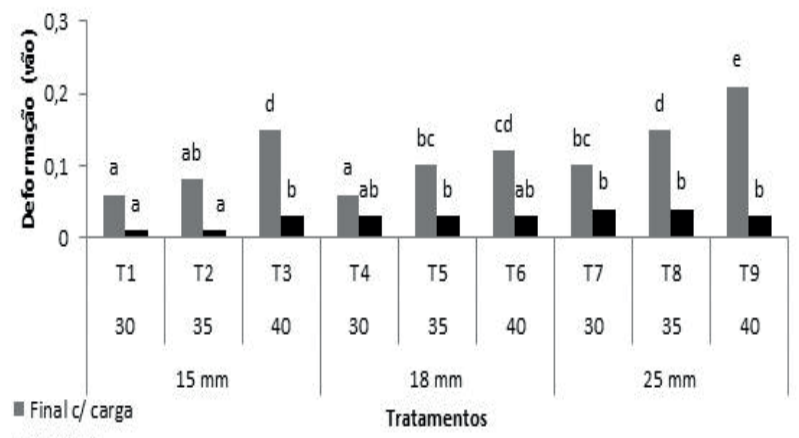

- Residual

Letras distintas significam diferenças significativas no teste de Tukey a 5\% de significância.

Figura 8 Valores médios da deformação final com carga e deformação residual relativos ao vão para os tratamentos com MDP.

Figure 8 Mean values of final deformation under load and residual deformation for each span for the treatments with MDP.

\section{Efeito do vão e da espessura}

Os resultados médios relativos à espessura mostram a deformação final e residual dos painéis de $15 \mathrm{~mm}$, $18 \mathrm{~mm}$ e $25 \mathrm{~mm}$ (Figura 9). Os maiores valores médios das deformações finais ocorreram no vão de relação $\mathrm{L} / \mathrm{h}=40$, assim como a maior deformação residual. Contudo, a deformação residual dos painéis de $M D P$ na relação $L / h=40$ apresentou diferença significativa em relação aos outros vãos.

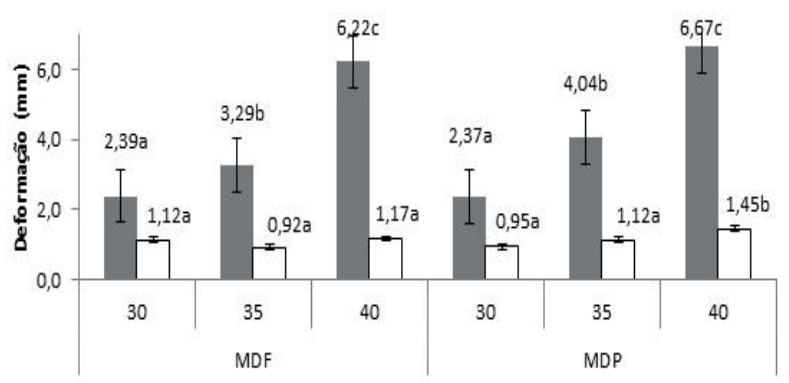

winal c/ carga

口Residual

Vão (L/h)

Letras distintas significam diferenças significativas no teste de Tukey a $5 \%$ de significância.

Figura 9 Valores médios da deformação em relação ao vão (relação L/h) dos painéis de MDF e MDP.

Figure 9 Mean values of deformation for span ( $\mathrm{L} / \mathrm{h}$ relationship) for MDF and MDP panels.

Analisando-se o efeito da espessura podese observar que o maior valor médio da deformação final com carga ocorreu para os painéis com $25 \mathrm{~mm}$ de espessura. Entretanto, o maior valor médio da deformação residual ocorreu para os painéis de MDF com $15 \mathrm{~mm}$ de espessura, (Figura 10). Apesar disso, deve-se considerar que os corpos-de-prova de $25 \mathrm{~mm}$ também possuíam os maiores vãos, apesar de terem a mesma relação $\mathrm{L} / \mathrm{h}$.

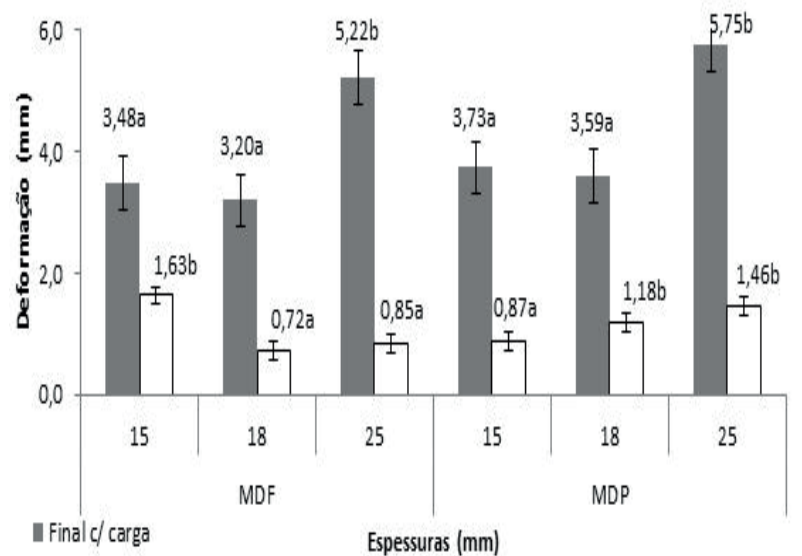

पResidual

Letras distintas significam diferenças significativas no teste de Tukey a $5 \%$ de significância.

Figura 10 Valores médios da deformação em relação a espessura dos painéis de MDF e MDP.

Figure 10 Mean values of deformation for thickness of the MDF and MDP panels.

\section{CONCLUSÃO}

Nos ensaios realizados conclui-se que:

a) Vãos menores apresentam menores deformações, independente da espessura utilizada. Portanto entre os vãos avaliados, a melhor relação encontrada foi $L / h=30$.

b) Painéis com maiores valores de MOE foram mais resistentes à deformação.

c) Os resultados do MDF foram ligeiramente superiores aos do MDP nas deformações com carga, no entanto, na deformação residual ambos apresentaram valores semelhantes.

d) O dimensionamento do vão é o requisito mais importante para se obter produtos mais resistentes à deformação.

\section{REFERÊNCIAS}

AMERICAN SOCIETY FOR TESTING AND MATERIALS DI037-93, Standard methods of evaluating the properties of wood-base fiber and particle panel materials. West Conshohocken, 1995.

ASSOCIAÇÃO BRASILEIRA DE NORMAS TÉCNICAS, ABNT NBR I396I, Móveis para escritório - Armários. Rio de Janeiro, 2010. 
BODIG, J., Jayne, B.A., Mechanics of Wood and Wood Composites. Malabar: Krieger Publishing Co, 1993.

ELEOTÉRIO, R. J. Propriedades Físicas e Mecânicas de Painéis de MDF de Diferentes Densidades e Teores de Resina. 2004. 122 p. Dissertação (Mestrado em Ciencia e Tecnologia de Madeiras) - Universidade de São Paulo, Piracicaba.

FRIHART, Ch R. Handbook of wood chemistry and wood composites. Rowell, R., Ed, 2005.
GREEN D. W., et al, Wood Handbook, Chapter 04: Mechanical Properties of Wood, Madison: Government Printing Office, 1987.

MALONEY, T. M., Modern particleboard and dry-process fiberboard manufacturing. San Francisco: Miller Freeman, 1993.

MORESCHI, J. C., Propriedades Tecnológicas da Madeira. Curitiba: UFPR, 2005.

DA ROSA, S. E. S., et al, O Setor de Móveis na Atualidade: uma Análise Preliminar. Rio de Janeiro Boletim Móveis. BNDES, 2007. 
